\title{
Allergen immunotherapy for IgE-mediated food allergy: protocol for a systematic review
}

\author{
Sangeeta Dhami ${ }^{1 *}$, Ulugbek Nurmatov², Giovanni Battista Pajno ${ }^{3}$, Montserrat Fernandez-Rivas $^{4}$, \\ Antonella Muraro ${ }^{5}$, Graham Roberts ${ }^{6,7}$, Cezmi Akdis $^{8}$, Montserrat Alvaro-Lozano ${ }^{9}$, Kirsten Beyer ${ }^{10,11}$, \\ Carsten Bindslev-Jensen ${ }^{12}$, Wesley Burks ${ }^{13}$, George du Toit ${ }^{14}$, Motohiro Ebisawa ${ }^{15}$, Philippe Eigenmann ${ }^{16}$, \\ Edward Knol ${ }^{17,18}$, Mika Makela ${ }^{19}$, Kari Christine Nadeau ${ }^{20}$, Liam O'Mahony ${ }^{21}$, Nikolaos Papadopoulos ${ }^{22}$, \\ Lars Poulsen ${ }^{23}$, Cansin Sackesen ${ }^{24}$, Hugh Sampson ${ }^{25}$, Alexandra Santos ${ }^{26}$, Ronald van Ree ${ }^{27}$, \\ Frans Timmermans ${ }^{28}$ and Aziz Sheikh ${ }^{29}$
}

\begin{abstract}
Background: The European Academy of Allergy and Clinical Immunology (EAACI) is in the process of developing the EAACI Guidelines for Allergen Immunotherapy (AIT) for IgE-mediated food allergy. We seek to critically assess the effectiveness, cost-effectiveness and safety of AIT in IgE-mediated food allergy.

Methods: We will undertake a systematic review, which will involve searching international biomedical databases for published, in progress and unpublished evidence. Studies will be independently screened against pre-defined eligibility criteria and critically appraised using established instruments. Data will be descriptively and, if possible and appropriate, quantitatively synthesised.
\end{abstract}

Discussion: The findings from this review will be used to inform the development of recommendations for EAACI's Guidelines on AIT.

Keywords: Allergy, Allergen immunotherapy, Food allergy, Therapy, Sensitisation

\section{Background}

Food allergy is responsible for considerable morbidity and, in some cases, mortality [1]. Epidemiological studies have demonstrated that the prevalence and severity of food allergy may be increasing, particularly in children [2-5]. Food allergies can be divided into IgE-mediated acute allergic reactions manifesting as urticaria, vomiting, wheezing and anaphylaxis, and non-IgE-mediated food allergy which refers to delayed, cell-mediated reactions. This review is focused on IgE-mediated reactions.

Food allergies can be associated with significant reduction in quality of life, both of individuals who suffer from food allergy and their family members [6]. At present,

*Correspondence: sangeetadhami@hotmail.com

${ }^{1}$ Evidence-Based Health Care Ltd, Edinburgh, UK

Full list of author information is available at the end of the article avoidance measures are the cornerstone of management [7]. Difficulties in avoiding responsible food allergens can result in accidental exposure and the risk of triggering potentially life-threatening anaphylaxis [8]. Many individuals with food allergy therefore need to carry adrenaline (epinephrine) auto-injectors in order to selfmanage anaphylaxis reactions. This approach is however perceived as restrictive and still leaves patients at risk if accidental exposure occurs.

Alternative approaches are therefore being investigated. In particular, there is considerable international interest in the role of immunotherapy, which involves repeated administration of very small, but gradually increasing doses of the antigens to which individuals are allergic in the hope of allowing safe exposure to the foods in question. Allergen immunotherapy (AIT) has, 
for example, over the last century become established clinical practice in relation to the treatment of severe pollen, insect venom and drug allergy [9]. However AIT has yet to become established in the routine management of food allergy.

The European Academy of Allergy and Clinical Immunology (EAACI) is in the process of developing the EAACI Guidelines for AIT, and this systematic review is one of five inter-linked evidence syntheses that are being undertaken in order to provide a state-of-the-art synopsis of the current evidence base in relation to evaluating AIT for the treatment of food allergy, allergic rhinoconjunctivitis, venom allergy and allergic asthma, and allergy prevention, which will be used to inform the formulation of key clinical recommendations. The focus of this review, which builds on our previous related reviews $[10,11]$, is on assessing the effectiveness, safety and costeffectiveness of AIT in the management of IgE-mediated food allergy.

\section{Methods}

\section{Search strategy}

A highly sensitive search strategy has been developed, and validated study design filters will be applied to retrieve articles pertaining to the use of AIT for IgEmediated food allergy from electronic bibliographic databases. We have conceptualized the search to incorporate the four elements shown in Fig. 1.

To retrieve randomized controlled trials (RCTs), we will apply the Cochrane highly sensitive search strategy for identifying RCTs in MEDLINE [12]. To retrieve nonrandomised studies, i.e. controlled clinical trials (CCT) and quasi-RCTs, we will use the Cochrane Effective
Practice and Organisation of Care (EPOC) filter Version 2.4, available on request from the EPOC Group [13, 14]. To retrieve case series, we will use the filter developed by librarians at Clinical Evidence: http://clinicalevidence. bmj.com/x/set/static/ebm/learn/665076.html.

We will search the following databases:

- Cochrane Library including the:

- CENTRAL (Trials)

- Methods Studies

- Health Technology Assessments (HTA)

- Economic Evaluations Database (EED)

- MEDLINE (OVID)

- Embase (OVID)

- CINAHL (Ebscohost)

- ISI Web of Science (Thomson Web of Knowledge)

- TRIP Database (www.tripdatabase.com)

- Clinicaltrials.gov (NIH web).

- Current controlled trials (www.controlled-trials.com)

- Australian and New Zealand Clinical Trials Registry (http://www.anzctr.org.au)

The search strategy has been developed on OVID MEDLINE and then adapted for the other databases (see Additional file 1: Appendix). In all cases, the databases will be searched from inception to March 31, 2016. Additional references will be located through searching the references cited by the identified studies, and unpublished work and research in progress will be identified through discussion with experts in the field. We will invite experts who are active in the field from a range of disciplines and regions to add to the list of included
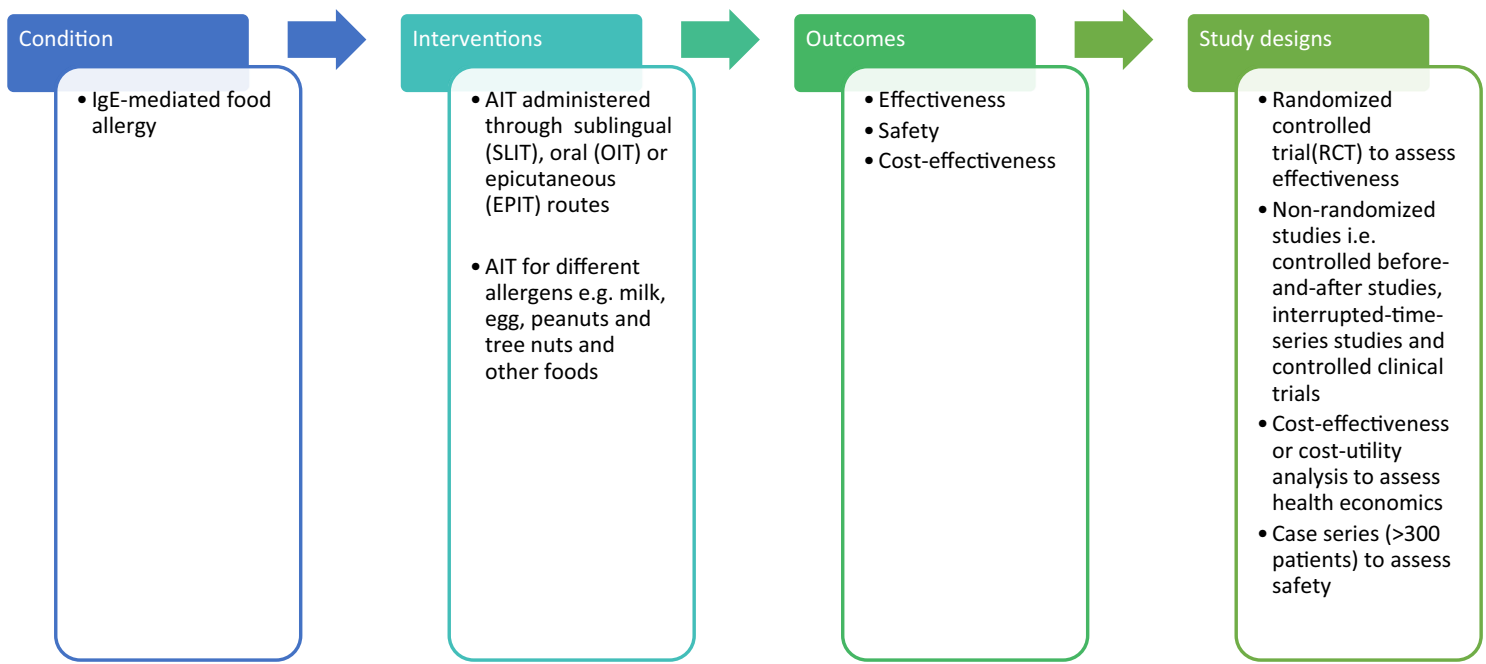

Fig. 1 Conceptualization of systematic review of allergen immunotherapy for IgE mediated food allergy 
studies by identifying additional published and unpublished papers and grey literature they are aware of and research in progress. We also, will search Web of Science to find published conference papers and all three major clinical trials repositories [Clinicaltrials.gov (NIH web); Current controlled trials (www.controlled-trials. com); Australian and New Zealand Clinical Trials Registry (http://www.anzctr.org.au) to identify trials in progress]. There will be no language restrictions employed; where possible, relevant literature will be translated into English.

\section{Inclusion criteria}

\section{Patient characteristics}

We will focus on studies conducted on patients of any age with a physician confirmed diagnosis of IgE-mediated food allergy to milk, eggs, peanuts, tree nuts and other foods in which there is also confirmation of allergic status through positive skin prick tests, specific-IgE or food challenge tests.

\section{Interventions of interest}

This review is focused on AIT for different allergens, i.e. milk, eggs, tree nuts, peanuts and other foods, administered through the following routes: oral, sublingual and epicutaneous.

\section{Comparators}

We are interested in studies comparing food allergy AIT with placebo or routine care (i.e. adrenaline autoinjector with or without antihistamines) or no treatment.

\section{Study designs}

RCTs, will be used to investigate effectiveness (i.e. desensitization and tolerance) and impact on disease specific quality of life; health economic analysis will be used to assess cost-effectiveness; and RCTs and case series with a minimum of 300 patients will be used to assess safety. We will appraise the evidence by looking at higher levels of evidence such as individual RCTs. However, given the likelihood that we will find only a limited number of RCTs, we will also search for and include the following non-randomized studies (NRS): controlled beforeand-after studies, interrupted-time-series studies and controlled clinical trials. Given the high inherent risk of bias in making inferences from such NRS, we will be very careful in making inferences from these data [15].

\section{Study outcomes}

- Primary Desensitization (i.e. the ability to safely consume foods containing the allergen in question while on AIT) or tolerance (the ability to consume foods containing the allergen in question after dis- continuing AIT) at food challenge, as defined in the relevant studies.

- Assessment of changes in disease specific quality of life using a validated instrument; in making this assessment we will focus on the minimal clinically important difference change in quality of life [16].

- Secondary Safety as assessed by local and systemic reactions in accordance with the World Allergy Organization's grading system of side-effects $[17,18]$.

- Health economic analysis from the perspective of the health system/payer as reported in studies.

\section{Exclusion criteria}

The following exclusion criteria will be applied:

- Reviews, systematic reviews, discussion papers, nonresearch letters and editorials

- Animal studies

- Quantitative studies not employing systematic review or RCT, or employing NRS designs other than those detailed above

- Qualitative studies

- Case series (of $<300$ patients).

\section{Study selection}

All references will be uploaded into the systematic review software Distiller and undergo initial deduplication. Study titles and abstracts will be independently checked by two reviewers according to the above selection criteria and categorized as: included, not included or unsure. Calibration will be undertaken after the first 50 screens to review any discrepancies between reviewers. For those papers in the unsure category, we will retrieve the fulltext and re-categorize as above. Any discrepancies will be resolved through discussion and, if necessary, a third reviewer will be consulted. Full text copies of potentially relevant studies will be obtained and their eligibility for inclusion independently assessed. Studies that do not fulfil all of the inclusion criteria will be excluded.

\section{Quality assessment strategy}

Quality assessments will independently be carried out on each study by two reviewers using the relevant quality assessment tools. Health economic studies will be assessed using the relevant CASP tool for economic evaluations [19]. RCTs, quasi-RCTs and CCTs will be assessed for generation of allocation sequence, concealment of allocation, baseline outcome measurements, baseline characteristics, incomplete outcome data, blinding of outcome assessor, protection against contamination, selective outcome reporting and other risks of bias. The Cochrane Risk of Bias tool will be used for RCTs and the Cochrane ACROBAT tool will be used for NRS 
[12]. Similarly, we will use the quality assessment form produced by the National Institute for Health and Clinical Excellence (NICE) to critically appraise case series [20]. Any discrepancies will be resolved by discussion or, if agreement cannot be reached, a third reviewer will arbitrate

\section{Data extraction, analysis and synthesis}

Data will be independently extracted onto a customized data extraction sheet in Distiller by two reviewers, and any discrepancies will be resolved by discussion or, if agreement cannot be reached, by arbitration by a third reviewer.

A descriptive summary with summary data tables will be produced to summarize the literature. If clinically and statistically appropriate, meta-analysis will be undertaken using random-effects modeling given the known clinical heterogeneity between studies. In the event of finding significant statistical heterogeneity between studies (assessed using $\mathrm{I}^{2}$ ), this will initially be visually inspected and then, if appropriate, be investigated through the prespecified subgroup and sensitivity analyses (see below). We will preferentially report on RRs with 95 \% CIs A narrative synthesis of the data will also be undertaken.

\section{Sensitivity and subgroup analyses, and assessment for publication bias}

Sensitivity analyses will be undertaken by comparing the findings between RCTs and NRS, and by comparing the results from studies that have employed double-blind placebo controlled food challenges versus those using other outcomes to assess for desensitization and/or tolerance.

Subgroup analyses will be undertaken to compare:

- Children (5-11 years) versus adolescents (1217 years) versus adults ( $\geq 18$ years)

- Treatment duration: $<3$ versus $\geq 3$ years

- Years of follow up: end of treatment, 2 versus $\geq 2$ years

- Route of administration: e.g. SCIT versus SLIT

- Allergens used for AIT

- Severity of food allergy: mild/moderate versus severe

- Primary versus secondary IgE-mediated food allergy.

Where possible, publication bias will be assessed through the creation of funnel plots, and tested by Egger's regression test and Begg's rank correlation test [21, 22].

\section{Registration and reporting}

This review will be registered with the International Prospective Register of Systematic Reviews (PROSPERO): http://www.crd.york.ac.uk/prospero/. The Preferred Reporting Items for Systematic Reviews and
Meta-Analyses (PRISMA) checklist will be used to guide the reporting of the systematic review: http://www. prisma-statement.org/.

\section{Discussion}

This review will involve systematically identifying, critiquing and synthesizing the evidence on the effectiveness, cost-effectiveness and safety of AIT for the management of IgE mediated food allergy. It will build on earlier reviews in this area $[10,11]$. The findings from this review will be used to inform the development of recommendations for EAACI's Guidelines on AIT. We anticipate that this review will report in 2016.

\section{Additional file}

Additional file 1. Appendix 1: Search strategy.

\section{Authors' contributions}

This protocol was drafted by SD and UN and the search strategy was developed by UN. It was revised following critical review by AS and then revised by all co-authors. All authors read and approved the final manuscript.

\section{Author details}

1 Evidence-Based Health Care Ltd, Edinburgh, UK. ${ }^{2}$ Division of Population Medicine Neuadd Meirionnydd, School of Medicine, Cardiff University, Heath Park, Cardiff, UK. ${ }^{3}$ Allergy Unit, Department of Pediatric, University of Messina, Messina, Italy. ${ }^{4}$ Allergy Department, Hospital Clínico San Carlos, IdISSC, Madrid, Spain. ${ }^{5}$ Food Allergy Referral Centre Veneto Region, Department of Women and Child Health, Padua General University Hospital, Padua, Italy. ${ }^{6}$ The David Hide Asthma and Allergy Research Centre, St Mary's Hospital, Newport Isle of Wight, NIHR Respiratory Biomedical Research Unit, University Hospital Southampton NHS Foundation Trust, Southampton, UK. ${ }^{7}$ Faculty of Medicine, University of Southampton, Southampton, UK. ${ }^{8}$ Swiss Institute for Allergy and Asthma Research, Davos, Switzerland. ${ }^{9}$ Paediatric Allergy and Clinical Immunology Section, Hospital Sant Joan de Déu, Universitat de Barcelona, Barcelona, Spain. ${ }^{10}$ Pediatric Pneumology and Immunology, Charité Universitätsmedizin, Berlin, Germany. ${ }^{11}$ Icahn School of Medicine at Mount Sinai, New York, NY, USA. ${ }^{12}$ Department of Dermatology and Allergy Centre, Odense University Hospital, Odense, Denmark. ${ }^{13}$ Department of Pediatrics, School of Medicine, University of North Carolina at Chapel Hill, Chapel Hill, NC, USA. ${ }^{14}$ Division of Asthma, Allergy and Lung Biology, Department of Paediatric Allergy, MRC and Asthma Centre in Allergic Mechanisms of Asthma, King's College London and St Thomas NHS Foundation Trust, London, UK. ${ }^{15}$ Department of Allergy, Clinical Research Center for Allergy and Rheumatology, Sagamihara National Hospital, Sagamihara, Kanagawa, Japan. ${ }^{16}$ University Hospitals of Geneva and Medical School, University of Geneva, Geneva, Switzerland. ${ }^{17}$ Department of Dermatology and Allergology, University Medical Center Utrecht, Utrecht, The Netherlands.

${ }^{18}$ Department of Immunology, University Medical Center Utrecht, Utrecht, The Netherlands. ${ }^{19}$ Skin and Allergy Hospital, Helsinki University Hospital, Helsinki, Finland. ${ }^{20}$ Division of Immunology, Allergy and Rheumatology, Department of Pediatrics, Stanford University, Stanford, CA, USA. ${ }^{21}$ Swiss Institute of Allergy and Asthma Research (SIAF), University of Zurich, Davos, Switzerland. ${ }^{22}$ Department of Allergy, 2nd Pediatric Clinic, University of Athens, Athens, Greece. ${ }^{23}$ Allergy Clinic, Copenhagen University Hospital, Gentofte, Denmark. ${ }^{24}$ Koç University Hospital, Istanbul, Turkey. ${ }^{25}$ World Allergy Organization (WAO), Milwaukee, WI, USA. ${ }^{26}$ Division of Asthma Allergy and Lung Biology, Department of Paediatric Allergy, King's College London/ Guy's, and St Thomas'Hospital NHS Foundation Trust, London, UK. ${ }^{27}$ Department of Otorhinolaryngology, Academic Medical Center, Amsterdam, The Netherlands. ${ }^{28}$ Nederlands Anafylaxis Netwerk - European Anaphylaxis Taskforce, Dordrecht, The Netherlands. ${ }^{29}$ Allergy and Respiratory Research Group, The University of Edinburgh, Edinburgh, UK. 


\section{Competing interests}

S. Dhami: support to undertake the systematic review; U. Nurmatov: support to undertake the review; Giovanni Pajno: none; Montserrat Fernandez-Rivas: Participation in immunotherapy clinical trials sponsored by ALK-Abello; A. Muraro: Acting in consulting capacity for ALK, Meda Pharma, Nestle, Nutricia, Novartis. Grants from: Nestlé: Co-investigator for research protocol, Nutricia: Co-investigator for research protocols; G. Roberts: Materials for research programme (ALK-Abello), research grant (ALK-Abello), advisory board (ALKAbello), speaker (Allergy Therapeutics, ALK-Abelo); C. Akdis: consulting fee Novartis, Boehringer-Ingelheim; stocks Davos Diagnostics, Allimentary Health Pharma Davos; research grant Novartis, Allergopharma; M. Alvaro-Lozano: none; K. Beyer: European Union, German Research Foundation,Berliner Sparkasse, Danone, ThermoFisher Diagnostics, Foundation for the Treatment of Peanut Allergy, author of A European perspective on immunotherapy for food allergies; C. Bindslev-Jensen: Grant from Hal Allergy. Consultancy for Hal Allergy; W Burks: none; G. du Toit: Equity in the FoodMaestro Application. Grants supporting the LEAP Study paid to Kings College, London. Author of the 2015 NEJM LEAP Study manuscripts that do not primarily deal with immunotherapy; M. Ebisawa: none; P. Eigenmann: Grants from LETI, Nestlé, ThermoFisher2, Consulting fee or honorarium from Danone, Novartis, ALK,DBV technologies, Stallergenes, Allergopharma; Edward Knol: Research grant for analysis modified peanut and cows milk allergens from HAL Allergy, Numico research. Consultancy fee from HAL Allergy, advice on peanut allergens; M. Makela: not known; K. Christine Nadeau: none; L. O'Mahony: Consultancy to Alimentary Health Ltd, a probiotic company; N. Papadopoulos: Grant from GSK, NESTLE, MERCK. Consulting fee from GSK, ABBVIE, Novartis, Menarini, Meda, AlK-ABELLO, Allergopharma, Uriach, Stallergenes. Payment for development of educational presentations for Abbvie, Sanofi, Menarini \& Meda; L. Poulsen: Grants for Clinical trials in collaboration with ALK, Stallergenes, BioMay, and Anergis; C. Sackesen: Funding from MSD to support laboratory tests for the study 'Effects of the montelukast therapy on asthma and allergic inflammation in children with food allergy'; H. Sampson: received grants or other research support from NIAID; National Institutes of Health, published an editorial accompanying LEAP study publication; A. Santos: none; R. van Ree: Consultancy for HAL Allergy; F. Timmermans: none with respect to this study; $A$. Sheikh: support to co-ordinate the undertaking of the systematic review and development of the guidelines.

\section{Funding}

EAACl.

Received: 19 January 2016 Accepted: 17 May 2016

Published online: 05 July 2016

\section{References}

1. Bock SA, Munoz-Furlong A, Sampson HA. Further fatalities caused by anaphylactic reactions to food, 2001-2006. J Allergy Clin Immunol. 2007;119:1016-8.

2. Sicherer SH, Sampson HA. Peanut allergy: emerging concepts and approaches for an apparent epidemic. J Allergy Clin Immunol. 2007;120:491-503.

3. Nwaru BI, Hickstein L, Panesar SS, Muraro A, Werfel T, Cardona V, et al. The epidemiology of food allergy in Europe: a systematic review and metaanalysis. Allergy. 2014;69:62-75.

4. Kotz D, Simpson CR, Sheikh A. Incidence, prevalence, and trends of general practitioner-recorded diagnosis of peanut allergy in England, 2001 to 2005. J Allergy Clin Immunol. 2011;127(623-30):e1.

5. Nwaru BI, Hickstein L, Panesar SS, Roberts G, Muraro A, Sheikh A, on behalf of The EAACI Food Allergy \& Anaphylaxis Guidelines Group. Prevalence of common food allergies in Europe: a systematic review and meta-analysis. Allergy. 2014;69:992-1007.

6. DunnGalvin A, Dubois AE, Flokstra-de Blok BM, Hourihane JO. The effects of food allergy on quality of life. Chem Immunol Allergy. 2015;101:235-52.
7. Muraro A, Werfel T, Hoffmann-Sommergruber K, Roberts G, Beyer K, Bindslev-Jensen $C$, et al. EAACI food allergy and anaphylaxis guidelines: diagnosis and management of food allergy. Allergy. 2014;69:1008-25.

8. Primeau MN, Kagan R, Joseph L, Lim H, Dufreshne C, Duffy C, et al. The psychological burden of peanut allergy as perceived by adults with peanut allergy and the parents of peanut allergic children. Clin Exp Allergy. 2000;30:1135-43.

9. Calderón MA, Boyle RJ, Penagos M, Sheikh A. Immunotherapy: the meta-analyses. What have we Learned? Immunol Allergy Clin N Am. 2011;31(2):159-73.

10. Nurmatov U, Devereux G, Worth A, Healy L, Sheikh A. Effectiveness and safety of orally administered immunotherapy for food allergies: a systematic review and meta-analysis. Br J Nutr. 2014;111:12-22.

11. Nurmatov U, Venderbosch I, Devereux G, Simons FE, Sheikh A. Allergenspecific oral immunotherapy for peanut allergy. Cochrane Database Syst Rev. 2012;9:CD009014.

12. Higgins JPT, Green S, editors. Cochrane handbook for systematic reviews of interventions. Version 5.1.0 [updated March 2011]. The Cochrane Collaboration, 2011. www.cochrane-handbook.org. Accessed on 3 Sept 2015.

13. EPOC Group. http://epoc.cochrane.org/literature-searching-systematic-reviews

14. Cochrane Effective Practice \& Organisation of Care (EPOC) Group: Personal communication Michelle Fiander, Information Specialist \& Trial Search Co-ordinator. Ottawa, Canada: EPOC; 2012.

15. Effective Practice and Organisation of Care Group. What study designs should be included in an EPOC review and what should they be called. http://epoc.cochrane.org/sites/epoc.cochrane.org/files/uploads/ EPOC\%20Study\%20Designs\%20About.pdf. Accessed on 9 Dec 2015.

16. Salvilla SA, Dubois AE, Flokstra-de Blok BM, Panesar SS, Worth A, Patel S, et al. Disease-specific health-related quality of life instruments for lgEmediated food allergy. Allergy. 2014;69(7):834-44.

17. Passalacqua G,Baena-Cagnani C, Bousquet J, Walter Canonica G, Casalet T, Cox L, Durham S, Larenas-Linnemann D, Ledford D, Pawankar R. Potter P, Rosario N, Wallace D, Lockey R. Grading local side effects of sublingual immunotherapy for respiratory allergy: Speaking the same language J Allergy Clin Immunol; 2013. http://www.jacionline.org/article/ S0091-6749(13)00528-9/pdf.

18. World Allergy Organization Subcutaneous Immunotherapy Systemic Reaction Grading System. https://www.aaaai.org/Aaaai/media/MediaLibrary/PDF\%20Documents/Immunotherapy\%20Forms/7b-World-AllergyOrganization-Systemic-Reaction-Grading-systemx.pdf.

19. CASP checklist for Economic evaluations. http://media.wix.com/ugd/dde d87_3b2bd5743feb4b1aaac6ebdd68771d3f.pdf. Last Accessed on 3 Sept 2015.

20. Higgins JPT, Green S. Cochrane handbook for systematic reviews of interventions. Version 5.0.2 (Chapter 11, Section 11).

21. Egger M, Davey Smith G, Schneider M, et al. Bias in meta-analysis detected by a simple, graphical test. BMJ. 1997;315:629-34.

22. Begg CB, Mazumdar M. Operating characteristics of a rank correlation test for publication bias. Biometrics. 1994;50:1088-101.

\section{Submit your next manuscript to BioMed Central and we will help you at every step:}

- We accept pre-submission inquiries

- Our selector tool helps you to find the most relevant journal

- We provide round the clock customer support

- Convenient online submission

- Thorough peer review

- Inclusion in PubMed and all major indexing services

- Maximum visibility for your research

Submit your manuscript at www.biomedcentral.com/submit 